\title{
Revealing the Bonding of Nitrogen Impurities in Monolayer Graphene
}

Juan Carlos Idrobo ${ }^{1}$, Cong $\mathrm{Su}^{2,3}, \mathrm{Ju} \mathrm{Li}^{2,4}$, and Jing Kong ${ }^{3,5}$

${ }^{1}$ Center for Nanophase Materials Science, Oak Ridge National Laboratory, Oak Ridge, USA

${ }^{2}$ Department of Nuclear Science and Engineering, Massachusetts Institute of Technology, Cambridge, USA

${ }^{3}$ Research Laboratory of Electronics (RLE), Massachusetts Institute of Technology, Cambridge, USA

${ }^{4}$ Department of Materials Science and Engineering, Massachusetts Institute of Technology, Cambridge, USA

${ }^{5}$ Electrical Engineering and Computer Science, Massachusetts Institute of Technology, Cambridge, USA

The bonding characteristics of nitrogen impurities in monolayer graphene have been investigated for over a lustrum [1], since controlling the nature of nitrogen bonding could potentially impart unique functionalization of the material for electronic applications, similar to that of doped silicon [2].

Early transmission electron microscopy (TEM) experiments showed that there is a charge redistribution associated with nitrogen impurities in graphene [1]. For example, a higher electronegativity of the N-C bonds in nitrogen-doped graphene, when compared to edges or point defects, has been observed by scanning tunneling microscopy (STM) [2]. However, neither experiment could directly reveal the actual bonding configuration of individual nitrogen impurities in graphene.

Scanning transmission electron microscopy (STEM) experiments, using atomically-resolved electron energy-loss spectroscopy (EELS), have shown that under the presence of nitrogen, the carbon fine structure changes with respect to that of graphene [3]. However, the carbon K-edge spectra acquired experimentally had a low signal-to-noise ratio, limiting a direct quantitative analysis of the $\sigma$ and $\pi$ peaks in the carbon K-edge, and how these peaks are affected by the presence of nitrogen in the lattice. Additionally, the EELS experiment did not show the K-edge fine-structure of the nitrogen impurity, as previously shown for the case of individual Si impurities in graphene $[4,5]$.

Here, we present recent EELS data of individual nitrogen impurities in monolayer graphene with high signal-to-noise ratio. The EEL spectra were obtained with the carbon and nitrogen K-edges simultaneously acquired, allowing for the direct identification of the bonding characteristics of both elements.

Figure 1 shows an example of a Z-contrast STEM image where a nitrogen impurity is observed in monolayer graphene, as well as the corresponding carbon and nitrogen K-edges fine structure. The experiments were performed in an aberration-corrected Nion UltraSTEM ${ }^{\mathrm{TM}} 100$, equipped with a cold field emission electron source and a corrector of third and fifth order aberrations, operating at an accelerating voltage of $60 \mathrm{kV}$ [6]. EEL spectra were collected using a Gatan Enfina spectrometer, with $0.3 \mathrm{eV} /$ channel dispersion, giving an energy resolution of $0.9 \mathrm{eV}$. The convergence semi-angle for the incident probe and the EELS collection semi-angle were $30 \mathrm{mrad}$ and $48 \mathrm{mrad}$, respectively.

In this talk, we will discuss in detail how nitrogen affects the bonding characteristics of the surrounding carbon atoms, as well as the effect of other impurities, such as $\mathrm{P}, \mathrm{Al}$ and $\mathrm{Si}$. We will also present our 
efforts towards observing EELS fine-structure changes for single impurities using a monochromated aberration-corrected STEM [7].

\section{References:}

[1] J. Meyer et al., Nature Materials 10 (2011) p. 209-215

[2] L. Zhao et al., Science 333 (2011) p. 999-1003.

[3] R.J. Nicholls et al., ACS Nano 7 (2013) p. 7145-7150.

[4] W. Zhou et al., Physical Review Letters 109 (2012) p. 206803.

[5] Q. M. Ramasse et al., Nano Letters 10 (2013) p. 4989-4995.

[6] O. L. Krivanek, et al., Ultramicroscopy 108, (2008). p. 179-195.

[7] Research supported by Oak Ridge National Laboratory's Center for Nanophase Materials Sciences, which is a U.S. Department of Energy Office of Science User Facility. We also acknowledge support by the Center for Excitonics, an Energy Frontier Research Center funded by the US Department of Energy, Office of Science, Basic Energy Sciences under award no. DE-SC0001088.
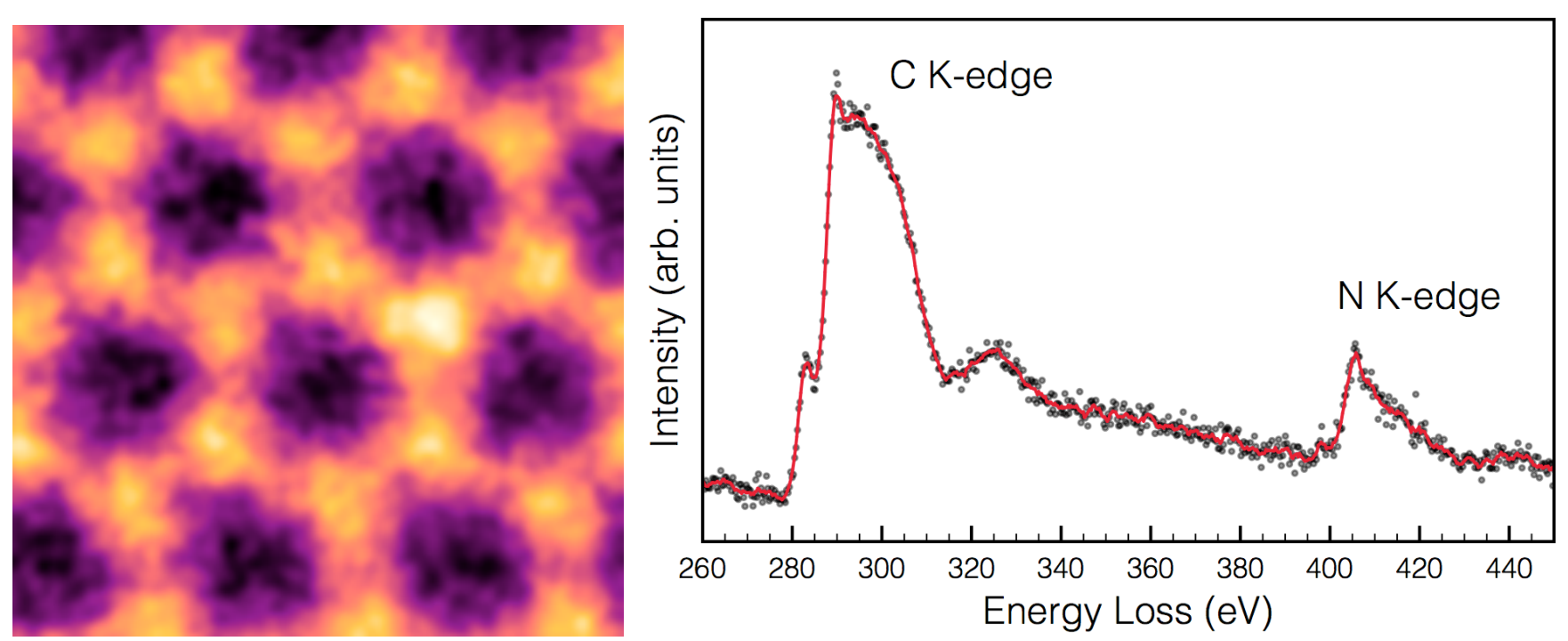

Figure 1. (Left) Z-contrast STEM image of an individual nitrogen impurity with three-fold carbon coordination in monolayer graphene (lighter color means higher intensity). The field of view of the image is $2 \times 2 \mathrm{~nm}^{2}$. (Right) Carbon and nitrogen K-edges obtained from a three-fold coordinated nitrogen impurity. 